\title{
OneGeology: from concept to reality
}
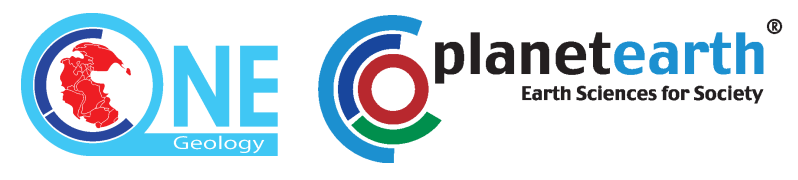

In the March 2007 edition of Episodes, an article described the origins of OneGeology and progress during its first 12 months. Since March 2007, the project has moved forward dramatically and in August 2008, at the 33rd International Geological Congress in Oslo, it was launched to the world.

The International Year of Planet Earth 2008 (IYPE) presented a great opportunity for geoscience, and for geological surveys in particular, to showcase their work. At the beginning of 2006 and with the potential stimulus of IYPE very much in mind, the germ of an idea was presented at short notice to the General Assembly of the Commission for the Geological Map of the World (CGMW) in Paris. Could we use this UN Year to begin the creation of an interoperable digital geological dataset of the planet at 1:1 million scale? Would it be possible to design and initiate a multi-lateral and multinational project that mobilised geological surveys, as part of an ongoing contribution, to act as the drivers and sustainable data providers of this global dataset? Could we synergistically use this vehicle of creating a tangible geological map to accelerate progress of an emerging global geoscience data model and interchange standard? Finally, could we use the project to transfer know-how to developing countries and reduce the length and expense of their learning curve, while at the same time producing maps and data that could attract interest and investment? These aspirations, plus the chance to generate a global digital geological dataset to assist in the understanding of global environmental problems and the opportunity to raise the profile of geoscience as part of IYPE, seemed more than enough reasons to take the proposal forward.

Throughout 2006 geologists and geological surveys around the world were canvassed for their views on this proposition and in the autumn of 2006 it was apparent that the concept was proving attractive to more than enough geological surveys and international bodies to organise a meeting to kick off the initiative; an initiative which had by now become known by the name "OneGeology". This kick-off meeting took place in March 2007, in Brighton, UK and at this meeting eighty-one participants from forty-three nations and fifty-three national and international bodies discussed and agreed the OneGeology aims and how best to achieve them. The workshop was a success and participants unanimously agreed a "Brighton Accord".
This Accord gave the OneGeology initiative the international backing it needed but just as importantly, OneGeology through the Brighton meeting and Accord, captured the imagination of the world's press and media and the story was taken up across the globe, increasing the profile of IYPE, the relevance of geoscience and placing OneGeology in the public eye.

OneGeology has a deceptively simple mission statement: A project to make webaccessible the best available geological map data worldwide at a scale of about 1:1 million, as a Geological Survey contribution to the International Year of Planet Earth 2008.

Within that it has three prime goals:

- improve the accessibility of geological map data,

- exchange know-how and skills so that all nations could participate, and

- accelerate interoperability in the geosciences and the take-up of a new "standard" (GeoSciML).

By the time of the 33rd International Geological Congress the project team had delivered the web map portal and the protocols, registries and technology to harvest and serve data from around the world. Second, they had produced guidance and provided support so that any geological survey could participate and serve their data. Third, the OneGeology team had accelerated and raised the profile of a crucial informatics standard: the geoscience mark-up Language GeoSciML. But in addition to these tremendous accomplishments, OneGeology had also achieved other things. It had contributed significantly to something that major global and regional bodies (including the United Nations and European Union) are desperately urgingthe creation of environmental spatial data infrastructures for planning and policy-making. At the 33rd IGC the project was endorsed by the directors of the geological surveys of the world and is providing a basis

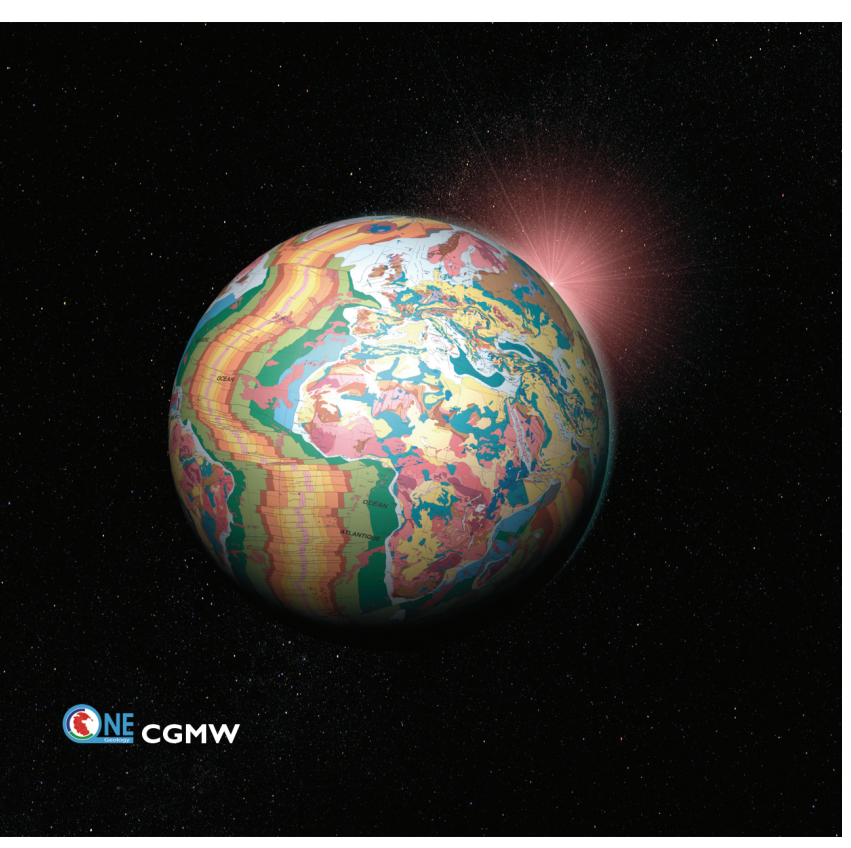

Geology on a 3D globe. for future collective and coherent action by them. Google references to One-Geology grew from 4,000 on 1 August 2008 to over 220,000 by mid-August. And when one looks more closely at some of these web pages, you see the way that improving access to the data has allowed many others to innovate; from developing new teaching resources for students, to web applications combining data and functionality from multiple sources. This outreach went well beyond the scientific and academic community and over 550 articles and broadcasts appeared in 10 days, from Nature to the BBC. All of these presented wonderful opportunities to describe to new audiences why geology is crucially important to society.

But who thinks OneGeology is important and why does it matter? Quite apart from the geological community, the majority of whom naturally agree that OneGeology is a "good thing", several other major stakeholders stand to benefit. These include: nations trying to attract inward investment (for example, Sierra Leone and Afghanistan); bodies who wish to effect planning and policy trans-nationally (for example, the United Nations and the European CommunityUNCLOS, UN Resolution 21, Spatial data Infrastructures, GEOSS, INSPIRE, GMES, SEIS); nations with cross-border issues (for example groundwater pollution in lower Rhine catchment; companies that operate multi-nationally (for example, mining, oil 


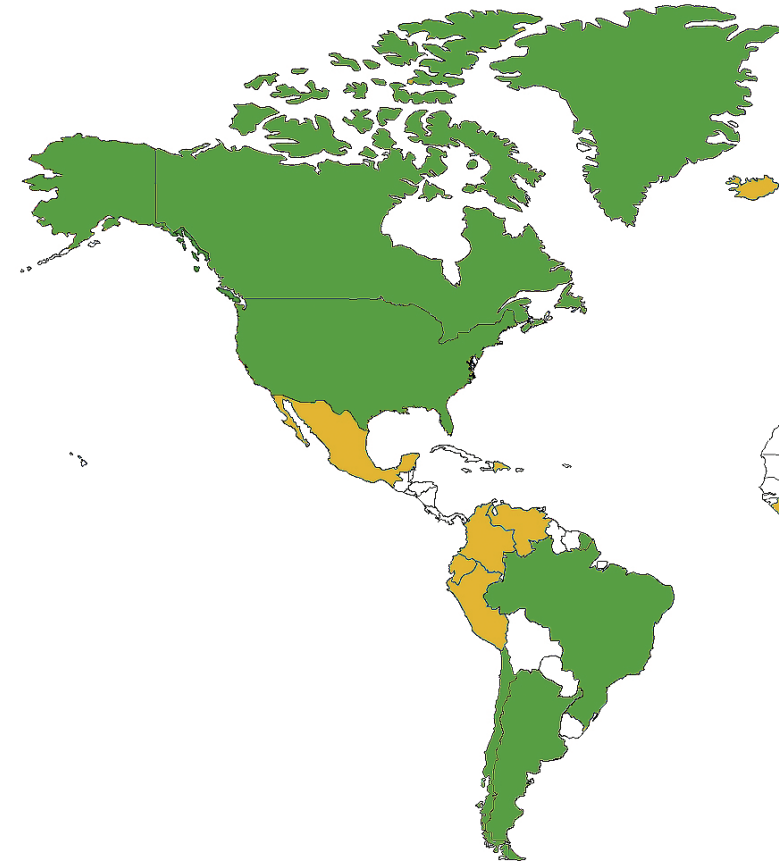

Country participating in OneGeology.

(1)

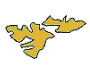

हnes
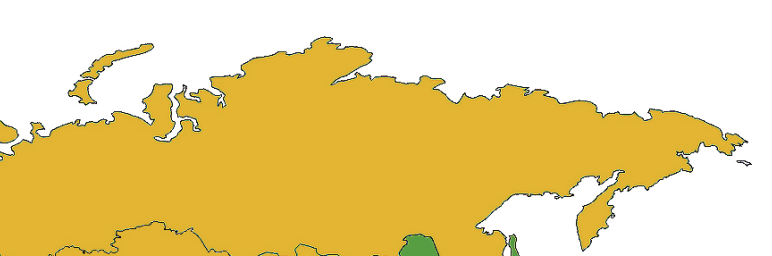

.

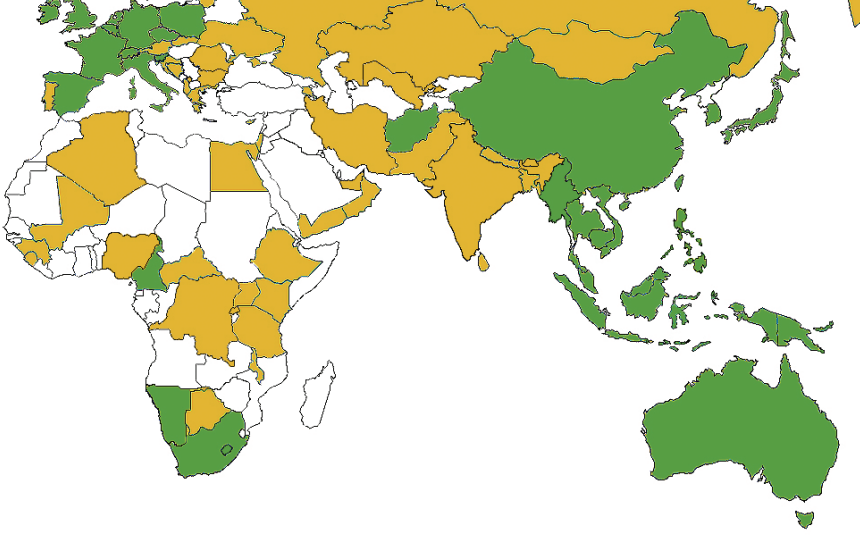

Current status map showing countries participating in OneGeology.

and gas, and insurance companies, and internet information brokers such as Google); those trying to understand regional impacts of global climate change (for example, holistic studies of the impacts of sea level rise or drought); and last but not least, nations who want to share and receive know-how.

At the beginning of the 33rd IGC, 81 nations were participating in OneGeology; by the end of the 33rd IGC the number had grown to 87 . Of the 81 nations, 30 were serving data to the OneGeology portal by 6 August 2008-this equated to almost 100 map datasets from national sources and also, importantly, from the prime international scientific body in global geoscience mapping, the CGMW. The technology to achieve OneGeology is not complex, but it in terms of the scale of the deployment it is truly world leading. A basic principle of OneGeology is that it must be open to all geological surveys to participate, regardless of development status and the project has devised protocols and systems to ensure this. OneGeology is thus open to those who currently possess only traditional paper geological maps, and to those operating sophisticated web mapping systems. The end-user does not require specialist software, only access to the Internet via a web browser. In this first phase, OneGeology is delivering digital geological map data from participating nations using Web Map Services (WMS). This is a distributed, dynamic and sustainable model, which unlike Google Earth leaves the data where it is best looked after and updated; that is with the provider nations. Each survey either reg- isters its web service with the OneGeology Portal or works with a partner survey (a "buddy") to serve that data. OneGeology technology is compliant with the international Open Geospatial Consortium (OGC) Web Map Service standard. Geological surveys may use a variety of software (e.g. MapServer) to serve their data. The Portal displays the map data served by each country and provides users with the ability to zoom, pan, switch map data on and off, change its opacity and transfer it to Google Earth.

The OneGeology initiative has made tangible progress in other areas too. The European Commission, under its eContentplus programme, has agreed to fund a 2-year, $€ 3.25$ million, 19-nation project known as OneGeology-Europe. This will move OneGeology forward faster and allow developments in higher resolution and applied data too. In the USA the National Science Foundation is providing almost $\$ 700000$ for a similar initiative in the 50 US states - a Geoscience Information Network. These and other continental initiatives will be well linked to ensure complementarity of development and maximum synergy and benefit globally.

Whatever OneGeology has done and is doing today, it is an inescapable fact that making geological map data web accessible across the globe would have happened at some point in the future. What those who have made OneGeology succeed today have declared is that they want this achievement to be the legacy of this generation of geologists, not the next. There are, after all, no insur- mountable scientific and technical problems and thus no excuses.

In conclusion, in addition to thanking a truly international project team, without whose efforts OneGeology would not be possible, it is perhaps fitting to end this article about a project that is attempting to share data and know-how globally, with a quotation from Chinese Premier Wen Jiabao, which appeared in Episodes in 2007.

"Geological sciences and geological structures do not end at national boundaries. Working on the same planet, geologists need to communicate and share knowledge with each other, and to draw on each other's experiences"

\section{Ian Jackson}

British Geological Survey,

Nottingham,

$U K$

Web page: www.onegeology.org

E-mail:ij@bgs.ac.uk

Telephone: +441159363214 